\title{
Imaging of cerebrovascular complications of infection
}

\author{
Guiquan Shen ${ }^{1 \#}$, Xudong Shen ${ }^{2 \#}$, Wei Pu ${ }^{3}$, Gang Zhang ${ }^{4}$, Alexander Lerner ${ }^{5}$, Bo Gao ${ }^{1,4}$ \\ ${ }^{1}$ Department of Radiology, The Affiliated Hospital of Guizhou Medical University, Guiyang 550004, China; ${ }^{2}$ Department of Radiology, Enshi \\ Central Hospital, Enshi 445000, China; ${ }^{3}$ Department of Radiology, Guizhou Provincial People's Hospital, Guiyang 550002, China; ${ }^{4}$ Department \\ of Radiology, Yantai Yuhuangding Hospital, Yantai 264000, China; ${ }^{5}$ Department of Radiology, Keck School of Medicine, University of Southern \\ California, Los Angeles, California, USA
}

\#These authors contributed equally to this work.

Correspondence to: Bo Gao, MD. Department of Radiology, The Affiliated Hospital of Guizhou Medical University, Guiyang 550004, China. Email: gygb2004@163.com; Gang Zhang, BSc. Department of Radiology, Yantai Yuhuangding Hospital, Yantai 264000, China. Email: sweetheart2@163.com.

\begin{abstract}
Cerebrovascular complications may occur in infectious diseases in the setting of infective endocarditis, central nervous system (CNS) infections, systemic bacteremia and sepsis. Cerebrovascular complications of infections include vasculitis, mycotic aneurysms, and thrombophlebitis. Infectious vasculitis of the CNS may cause cerebral hemorrhage, infarction or ischemia. Ruptured aneurysms may endanger the patient's life. Infectious thrombophlebitis may cause intracranial pressure to increase and lead to cerebral hemorrhage. These cerebrovascular complications are associated with a poor prognosis and often cause irreversible neurological deficits. Cerebrovascular events secondary to infection are not easily distinguishable from the more common cerebral infarct and non-infectious vasculitis. In addition, the clinical manifestations of cerebrovascular complications of infections are non-specific and highly variable. Therefore, early imaging, antibiotics, and anticoagulation may be lifesaving the patient's life and prevent disability. The main focus of this article is to review imaging analysis of the cerebrovascular complications of infections and imaging features that help differentiate them from non-infectious vascular diseases.
\end{abstract}

Keywords: Central nervous system (CNS); imaging; infection; vasculitis

Submitted Oct 04, 2018. Accepted for publication Nov 19, 2018.

doi: 10.21037/qims.2018.11.08

View this article at: http://dx.doi.org/10.21037/qims.2018.11.08

\section{Introduction}

The clinical manifestations of cerebrovascular complications of infections may overlap with non-infectious vascular diseases, and may be difficult to diagnose only by clinical manifestations. Cerebrovascular complications are not uncommon in clinical practice. Cerebrovascular involvement is the most severe extra-cardiac complication of infective endocarditis (IE), with up to $20-55 \%$ of patients with leftsided IE developing ischemic or hemorrhagic lesions (1). IE is thus associated with high mortality and morbidity (2). Up to $66 \%$ of patients with pediatric tuberculous meningitis can be complicated by cerebral infarction (3).
Meningovascular syphilitic infection is present in $15-$ $23 \%$ of patients with neurosyphilis (4). In contrast, the cerebrovascular complications of central nervous system (CNS) infection such as cerebral ischemia and mycotic aneurysms may appear insignificant or asymptomatic in the early stages. Some of the clinical manifestations of these complications are non-specific, including headache, convulsion, septicemia, cerebral hemorrhage, cerebral infarction, etc. However, some complications are very serious, especially in the case of mycotic aneurysms, which may develop rapidly (5). Imaging examination can detect these vascular complications, guide the clinical treatment of such complications, and evaluate the efficacy the treatment. 


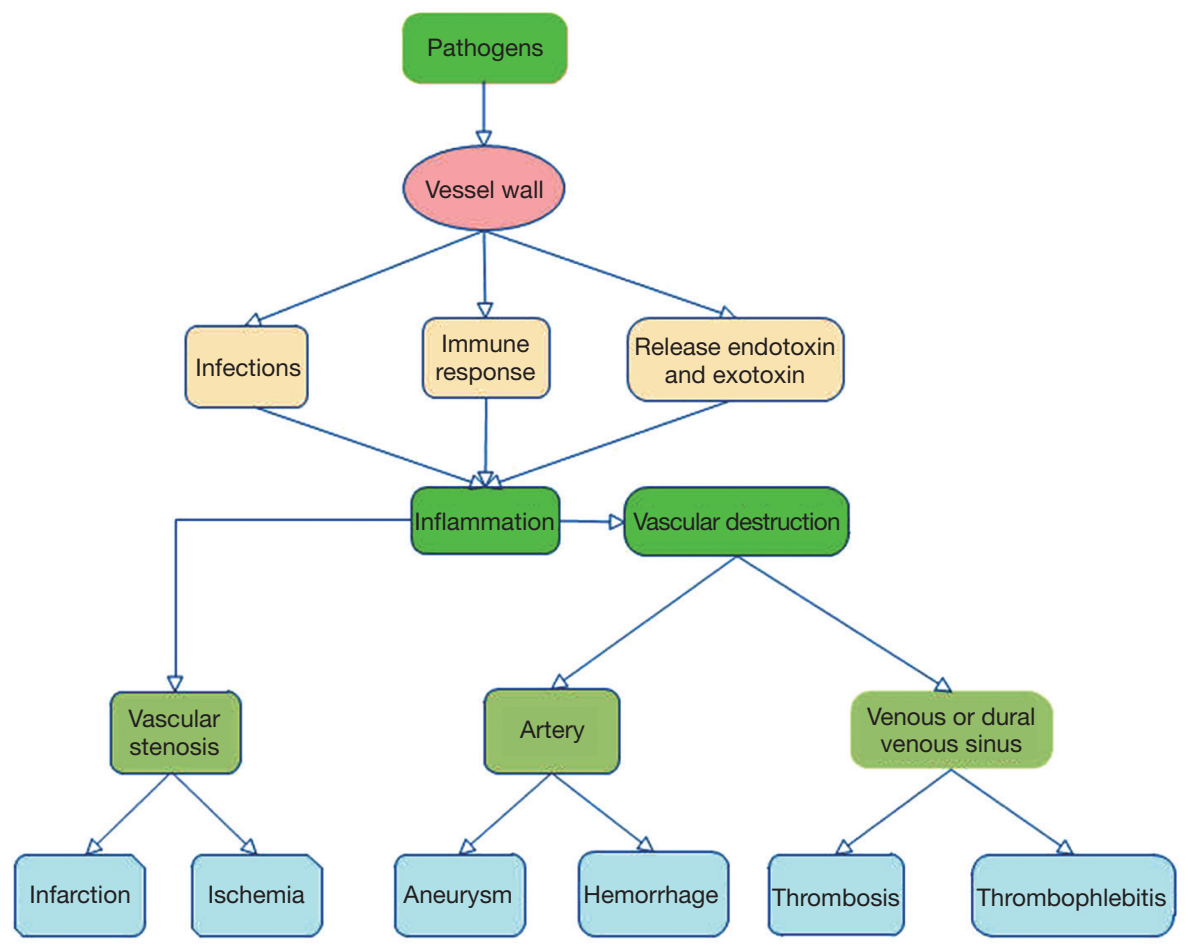

Figure 1 This flowchart illustrates the pathological changes of infectious central nervous system vasculitis. Direct damage and immune response to pathogens cause complications associated with infectious vasculitis of the central nervous system.

\section{Pathophysiology}

The infectious pathogens causing cerebrovascular complications include bacteria, fungi and viruses. The common pathogens include Mycobacterium tuberculosis, staphylococci, mucor fungi and human immunodeficiency virus (HIV). The pathogens can reach the vessels wall through different routes: via the endoluminal vascular space and through the endothelium, via the vasa vasorum, by direct extension from adjacent focus of infection, through direct inoculation during trauma or surgery, and by hematogenous contamination. Pathogens may enter via direct injury to the vascular endothelial cells, the media, and the adventitia through the above pathways. While in some cases the mechanism of injury is still unclear, many mechanisms have been proposed. Many infectious agents can trigger autoimmunity through various mechanisms (6). The microorganisms may induce changes in the host protein, causing the immune system to no longer recognize itself as "self" (7). Chronic infections can activate autoreactive B-cells and T-cells through different mechanisms and lead to vascular injury (8). These injury factors can cause inflammatory lesions in the intracranial blood vessels, and further spread inflammation to brain parenchyma. The inflammation of the cerebral artery with polymorphic neutrophils, enzymes and pro-inflammatory cytokines results in degradation of the layers of the vessel wall to a friable state and result in a defect within the wall leading to parenchymal, subarachnoid, intraventricular hemorrhages (9). Sustained inflammation mediated by the pathogens, $\mathrm{T}$ cells, macrophages and the pro-inflammatory cytokines leads to intimal thickening and vessel occlusion, ultimately leading to cerebral infarction or ischemia (10). In some cases, aneurisms are formed due to fragmentation and loss of the internal elastic lamina, intimal hyperplasia, medial fibrosis with muscle and elastic tissue loss (11). The venous or dural venous sinus involvement may lead to thrombosis and thrombophlebitis (12) (Figure 1).

\section{Infectious vasculitis or arteritis}

Vasculitis secondary to infection tends to have a poor prognosis. The most common manifestation of such vasculitis is ischemic infarct, with intracranial hemorrhage 

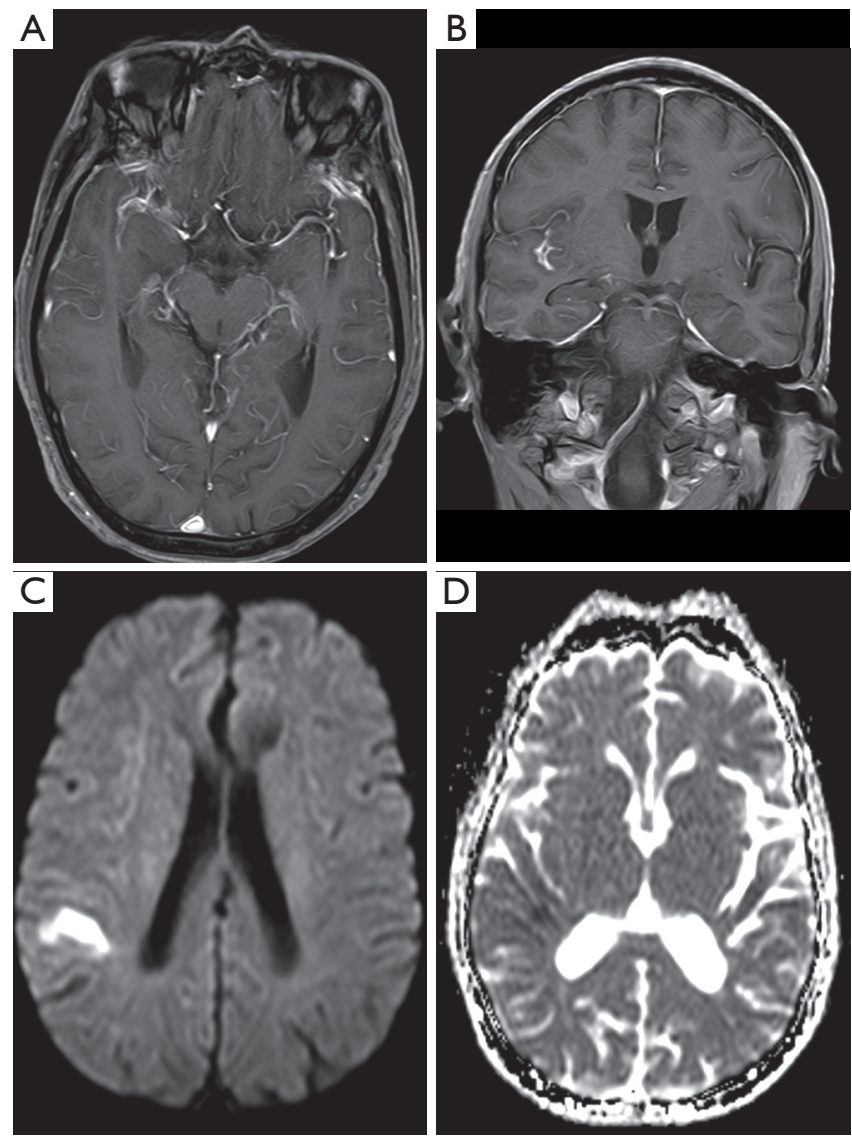

Figure 2 Tuberculous meningitis with vasculitis and cerebral infarction. Axial (A) and coronal (B) T1 post-contrast showed enhancement around the right middle cerebral artery. Axial DWI (C) and ADC map (D) showed infarction occurring in the vascular territory of the right middle cerebral artery. This suggests inflammatory stenosis or occlusion of the branches of the middle cerebral artery.

seen less frequently. The most common pathogens are Streptococcus pneumonia and Staphylococcus aureus, accounting for $67 \%$ and $21 \%$, respectively (13). A large number of studies have reported that parasitic and bacterial infections may cause severe infectious vasculitis and even moyamoya disease. Viral infections, especially patients with HIV infections may increase the risk of cerebral vascular accident (14). Left-sided IE-related CNS complications can be subclinical, asymptomatic events in up to $60-80 \%$ of cases (1). Studies have shown that the rapid treatment of bacterial meningitis with intravenous antibiotics and corticosteroids may reduce the risk of cerebral vasculitis complications (15). Unfortunately, infarction may present at any time, during or after completion of treatment (16).

CT and MRI examinations make an impact on the decision-making process regarding clinical management. Some indirect evidence of these complications can be found on non-contrast enhanced initial CT including cerebral infarction, skull base infection, brain abscess, hydrocephalus, etc. The most common imaging finding is cerebral infarction (Figure 2). Infarcts are often multifocal and may be found in the deep grey nuclei, thalami, and peripherally, predominantly at the grey-white junctions. They are often detected as multiple foci of acute cerebral infarction. The diffusion-weighted imaging (DWI) sequence in acute phase demonstrates hyperintensity (Figure 2). Less common findings include cerebral hemorrhage and subarachnoid hemorrhage (reported data varies from 1 to $9 \%)(4,17)$. The small foci of hemorrhage show as low signal on gradient-echo sequence (GRE) or susceptibilityweighted imaging (SWI).

Cerebral angiography has a relatively high detection rate for infectious vasculitis. Vascular inflammation is associated with thrombosis of and cerebral infarction. Vascular injury is a key factor in the mechanism of brain injury in bacterial meningitis (18). Angiography in vasculitis shows segmental vascular narrowing and dilatation, showing a "beaded appearance" similar to non-infectious vascular lesions. Chronic progressive stenosis or occlusion of the cerebral vessels, and abnormal vascular structures may be seen in the skull (Figure 3). Vessel wall imaging can be performed with high-resolution black-blood contrast enhanced $\mathrm{T} 1$ weighted MRI technique. In the active phase of inflammation, the walls of the affected intermediate and small arteries are thickened (Figure 4). The thickening is usually concentric (Figure 4), and less frequently eccentric (19). Gadolinium enhanced brain MRI has been proved to be very useful during follow-up. The diagnosis of cerebral vasculitis may be corroborated by decrease of gadolinium enhancement in the affected artery after corticosteroid treatment. MRI is helpful to monitor the inflammatory process and change the therapeutic strategy to prevent inflammatory reactivation by observing the degree of vascular enhancement (20).

\section{Infectious or mycotic intracranial aneurysm}

Infectious intracranial aneurysm (IIA) or mycotic aneurysm is defined as a focal dilatation of the cerebral artery vessels due to infection and secondary inflammation. IIA account for $0.7 \%$ to $6.5 \%$ of all intracranial aneurysms and up 



Figure $3 \mathrm{HIV}$-related vasculitis and aneurysms. (A) Axial head CT on a 7-year-old girl with left parietal lobe infarction. Angiography MRA (B) and DSA (C,D) showed bilateral segmental stenosis of internal carotid artery and multifocal large vessels fusiform aneurysmal. DSA showed multiple arterial occlusion changes and multiple arterial-arterial collaterals (such as involving bilateral posterior cerebral arteries). (The figure was provided by Fanny E. Morón MD, from the Department of Radiology, Baylor College of Medicine, Houston, Texas 77030, USA). MRA, magnetic resonance angiography; DSA, digital subtraction angiography; CT, computed tomography.

to $15 \%$ in children of the general population (21). IIA may be asymptomatic. A major complication of IIA is subarachnoid, parenchymal, or intraventricular hemorrhage secondary to aneurysm rupture. Ruptured IIA have a high mortality. The treatment of un-ruptured IIA is with broad spectrum intravenous antibiotics. However, IIA are also at risk of rupture during such therapy, and the risk of rupture of IIA during treatment was not reported to decrease (21). Therefore, it is necessary to perform imaging examinations during treatment. If the size of the IIA has decreased, interventional treatment is often not required. If the aneurysm size increases or new aneurysms are found during follow-up imaging, further intervention is required. The ruptured aneurysm requires immediate intervention, and endovascular treatment or open surgery is may be performed (22). 

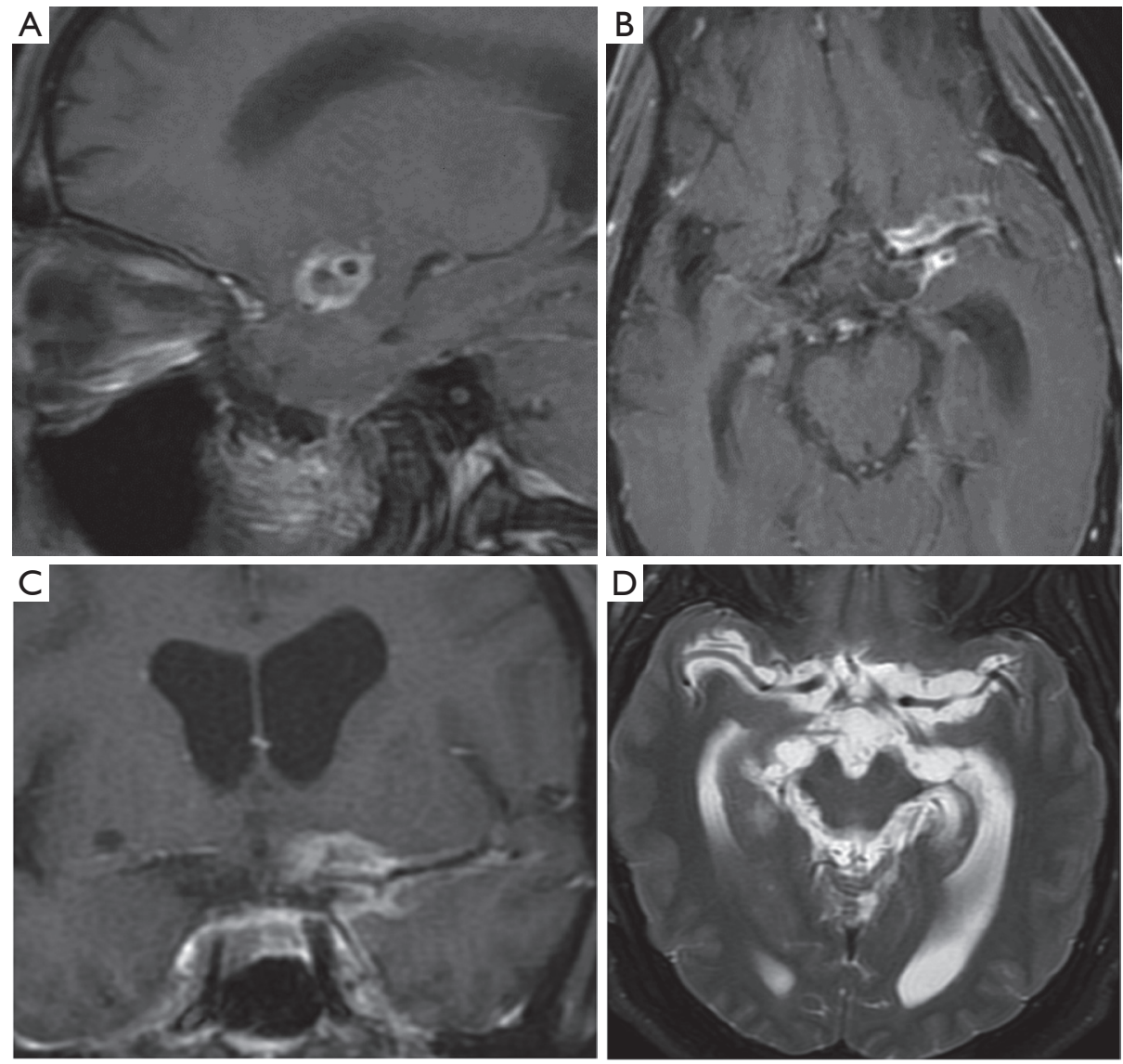

Figure 4 Neurocysticercosis with secondary inflammatory vasculitis. T1 post-contrast $(A, B, C)$ showed left Sylvian fissure enhancing cysts surrounding the left supraclinoid ICA-carotid terminus and proximal A1 and M1 segments of ACA and MCA (respectively) with circular enhancement around the vessels. Axial T2WI (D) revealed hydrocephalus. (The figure was provided by Fanny E. Morón MD, from the Department of Radiology, Baylor College of Medicine, Houston, Texas 77030, USA).

Non-contrast head CT is primarily used to assess whether patients with suspected cerebrovascular complications have subarachnoid hemorrhage, intraparenchymal hemorrhage or intraventricular hemorrhage (Figure 5). MRI is sensitive for intracranial lesions. MRI can detect signs of intracranial infectious lesions such as a focus of infection, cerebral edema, infarction, and pia mater enhancement (Figure 5). These findings are helpful in diagnosing infectious aneurysms. CT angiography (CTA) is superior to magnetic resonance angiography (MRA) for detection and evaluation of aneurysms. While CTA may adequately evaluate for aneurysms, digital subtraction angiography (DSA) may be performed in patients with negative or equivocal CTA, when IIA is strongly suspected. The imaging characteristics of mycotic aneurysms may include focal vascular dilatation, and saclike outpouching from the vessel wall (Figure 5).
The diagnosis of infectious aneurysm is supported by the following features: (I) the patient had a history of systemic or local infection, especially IE (23); (II) about $25 \%$ of mycotic aneurysms being multiple (24) (Figure 6), and up to $41 \%$ being saccular (25); (III) the neck of the aneurysm is frequently wide or absent (Figure 7). Rapid change in size or shape over time may occur, and new aneurysms may appear at the follow-up of angiographic imaging (26). Cho et al. (27) found that MRI can be used as a sensitive and specific tool to assess whether IE patients suffer from IIA. In their study, the following MRI imaging predictors for IIA were depicted: (I) contrast enhancement of microbleeds, (II) brain microbleeds $>5 \mathrm{~mm}$ or Sulcal SWI lesions, and (III) any MRI hemorrhage. The sensitivity of each group of IIA was $90 \%, 80 \%$, and $100 \%$, respectively. 

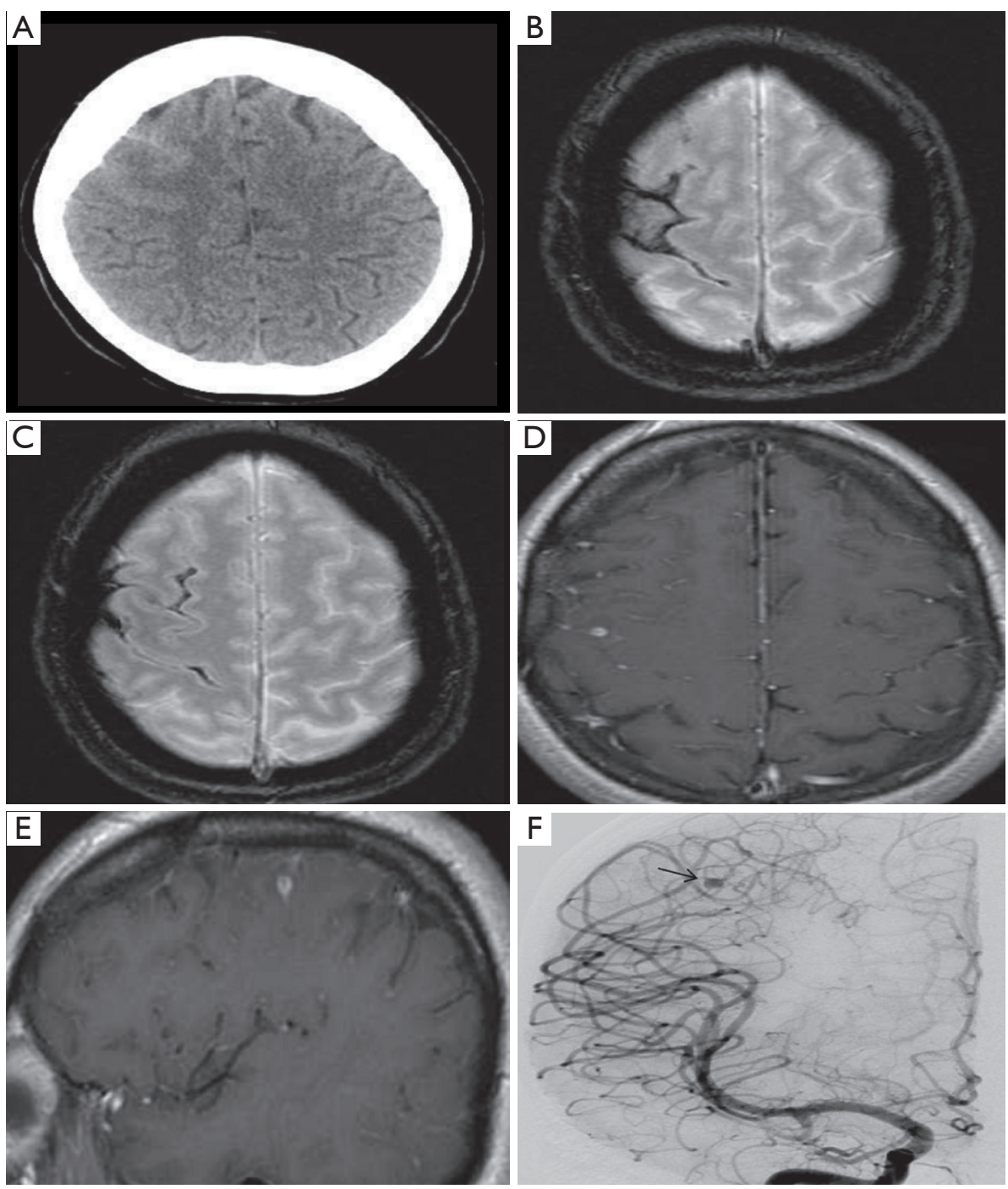

Figure 5 Aspergillosis in a leukemia patient with ruptured mycotic aneurysm. Axial non-contrast head CT (A) and axial T2* (B,C) showed right frontal subarachnoid hemorrhage. Post-contrast axial (D), sagittal (E) T1 showed small focus of pooling of contrast within the right middle frontal sulcus, and pial strengthening in the sulcus. DSA (F) confirmed that the right middle cerebral artery (M4) lesion was an infectious aneurysm. (The figure was provided by Fanny E. Morón MD, from the Department of Radiology, Baylor College of Medicine, Houston, Texas 77030, USA). DSA, digital subtraction angiography.

\section{Venous cerebrovascular complications of infections}

Infectious cerebral thrombophlebitis occurs due to inflammation of the vein wall. Infections involving the veins and venous sinuses cause pleomorphic neutrophils, enzymes, and pro-inflammatory cytokines to act on the vessel wall making it more vulnerable and permeable. The venous structures become prone to thrombosis and thrombophlebitis. Cerebral infectious thrombophlebitis is rare. However, women of childbearing age are at high risk, and about $58.1 \%$ of infectious thrombophlebitis occurs in this age group (28). Cerebral venous thrombosis (CVT) accounts for nearly all intracranial thrombosis with incidence $0.5-1.0 \%$, but the incidence increases to as high as $5 \%$ of all strokes in the younger population $(29,30)$. Infectious thrombophlebitis of the transverse sinus and sigmoid sinus is closely related to middle ear papillary infection, and infection of the skull base, paranasal sinuses 



Figure 6 Infective endocarditis complicated with multiple infectious aneurysms. (A,B) showed aneurysms in the right middle cerebral artery (MCA), the M1 segment of the left MCA and the anterior communicating artery. (C,D,E,F) showed multiple stenosis of the left MCA, the left anterior cerebral artery, and the right internal carotid artery.

and middle ear infection. Cavernous sinus thrombophlebitis is associated with paranasal sinusitis, orbital infections, facial and periodontal infections, and septicemia with involvement of the internal jugular vein. The superior sagittal sinus thrombophlebitis is rare, usually caused by meningitis, subdural and epidural abscesses.
CVT is usually negative on non-contrast CT images, contrast enhanced CT can show the enhancement of the affected vessel wall, the density of sinus cavity is inhomogeneous, and the venous sinus may have a filling defect (Figures 8,9). Venous sinus thrombosis causes venous return obstruction and reduces cerebral blood flow 



Figure 7 Mucor infection associated aneurysm. Axial CTA (A,B) showed left intraorbital cellulitis and opacified left frontal and ethmoidal sinuses with dilatation and irregularity of the left supraclinoid internal carotid artery (ICA). DSA (C) showed left ICA stenosis and aneurysm, while DWI (D) found multiple intracerebral infarctions. After interventional therapy (E), the patient's symptoms worsened, and CT examination revealed a large area of cerebral infarction in the left cerebral hemisphere (F). (The figure is provided by Fanny E. Morón MD, from the Department of Radiology, Baylor College of Medicine, Houston, Texas 77030, USA). CT, computed tomography; CTA, CT angiography; DSA, digital subtraction angiography; DWI, diffusion-weighted imaging.

and cerebral perfusion pressure, resulting in an impaired blood-brain barrier. Infection and thrombosis affect cerebrospinal fluid circulation, leading to an increase in intracranial pressure. The increase in intracranial pressure is characterized by a widening of the subarachnoid space. MRI examination can detect intracranial infections. Thrombophlebitis is characterized by thickening of the vessel wall, thrombosis, and enhancement of the vessel wall (Figure 10). On gradient-echo and SWI, the thrombus appears mostly hypointense in acute, subacute and chronic phases of thrombosis (30). Enhanced CT venography (CTV) and magnetic resonance venography (MRV) are considered the most reliable examinations for the confirmation of the diagnosis of CVT. Enhanced CTV and MRV are considered to be the most reliable examinations to confirm the diagnosis of CVT. CTV and MRV can directly display venous sinus thrombosis, which is characterized by a filling defect in the sinus. CTV is better for the detection of thrombi in smaller sinuses and in detecting partial venous thrombosis (31). 

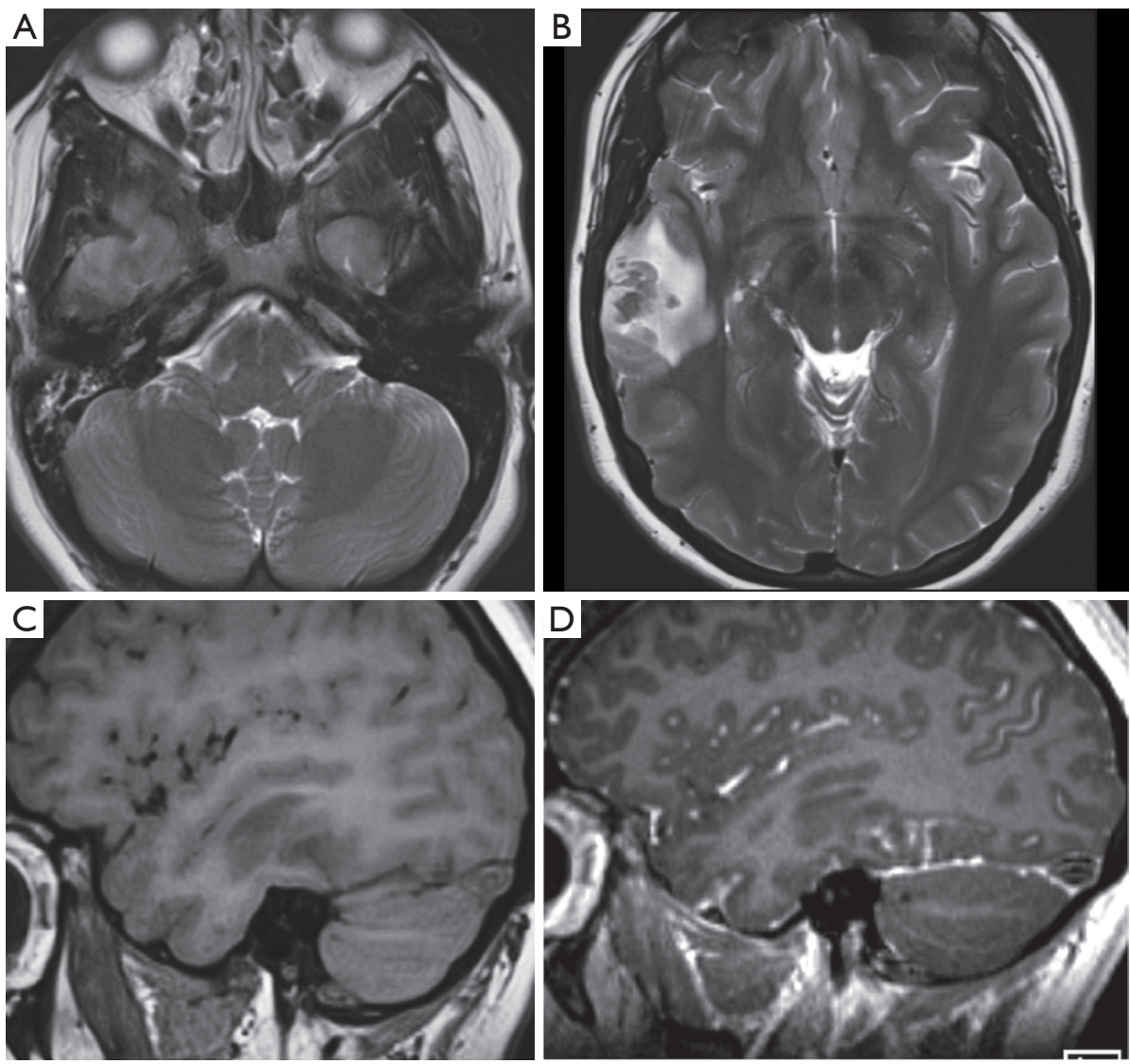

Figure 8 Left transverse-sigmoid (LTS) sinuses thrombophlebitis. Axial T2 (A,B) showed hyperintense opacification of the left petrous apex, left mastoid/middle otomastoiditis and abscess in the left cerebellum. Axial and coronal T1 post-contrast can be seen in (C,D). Sagittal T1 (C) showed T1 isointense filling on the right transverse-sigmoid sinus, while on post-contrast T1 (D) the "delta sign" corresponds to the filling defect and mild associated inferior temporal lobe leptomeningeal enhancement. (The figure is provided by Fanny E. Morón MD, from the Department of Radiology, Baylor College of Medicine, Houston, Texas 77030, USA).

\section{Differential diagnosis}

The vascular complications of infections primarily need to be differentiated from non-infectious vascular diseases. Vascular complications of infections are often found during treatment of infection or after a chronic infection. Infectious thrombophlebitis has a low clinical incidence, often secondary to severe infections around the skull base and sinus. Imaging findings supporting infectious vasculitis include: thickening of the vessel wall, enhancement, blurring of the edge of the vessel wall (Figure 2), and visualization of exudative lesions. Occurrences of non-infectious vasculitis usually have no history of infection, and most have systemic vascular diseases, and vascular wall enhancement and perivascular exudation being rare. Cerebral MRA, CTA and CT perfusion (32), pathological examination, and blood culture can help to confirm the diagnosis.

Imaging diagnosis of infectious vasculitis caused by different infectious pathogens is difficult, however, clinical findings are often valuable for differential diagnosis. Vascular complications caused by bacterial infection may occur in the acute phase of meningitis or several days to several weeks after the start of treatment and normalization of cerebrospinal fluid. Vascular complications of tuberculous meningitis are more common in the chronic phase than in acute tuberculous meningitis. Lenticulostriate and thalamoperforating arteries are the most commonly affected vessels and up to $75 \%$ of the tuberculosis related infarcts are located in the basal ganglia and thalamus (13). Patients infected with HIV have a higher risk of stroke than the general population, with most HIV-infected patients developing small-vessel vasculitis (16), which usually affects 

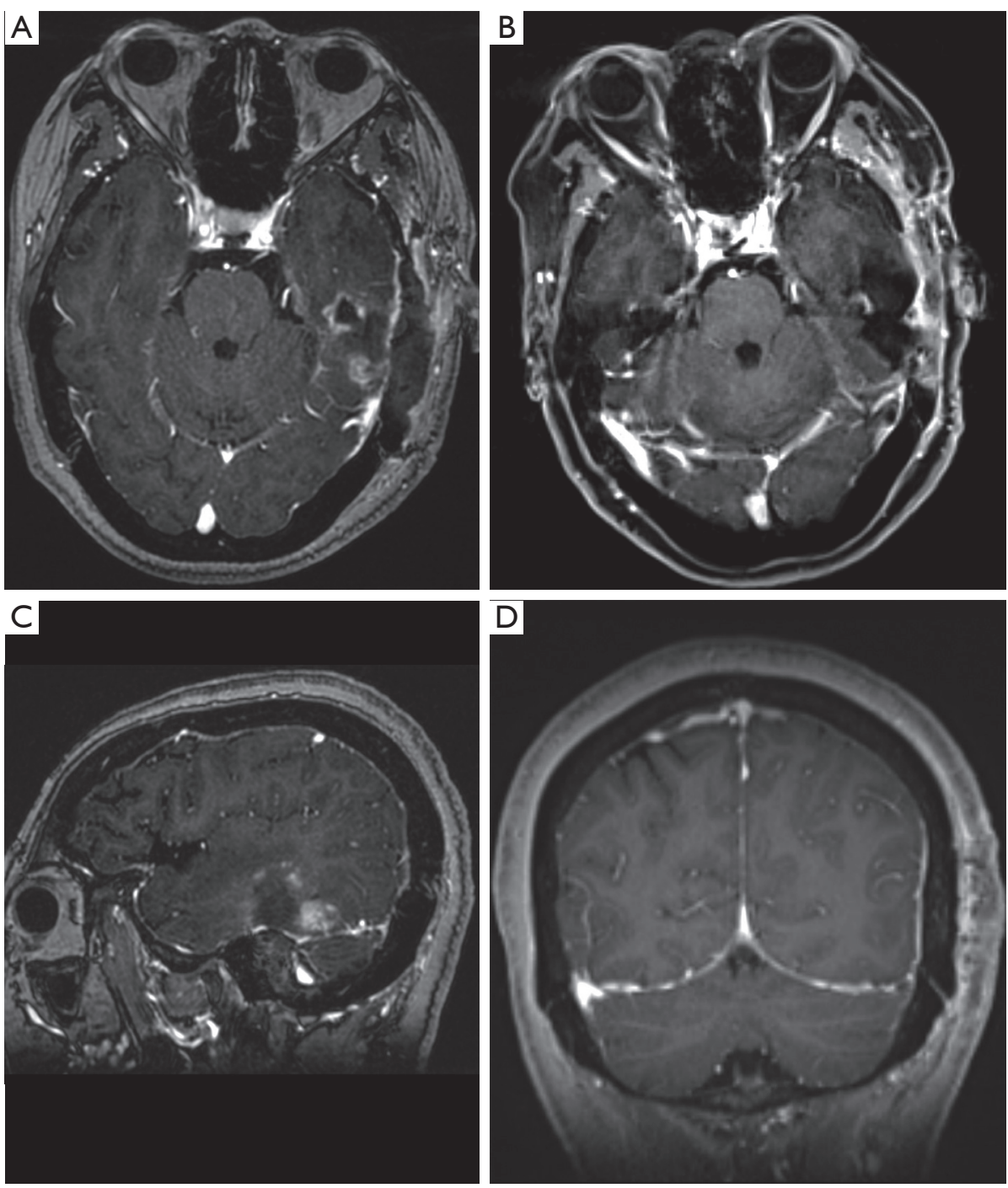

Figure 9 Cryptococcus infection with thrombophlebitis. Axial T1 post-contrast (A,B) showed thickening of the scalp on the left temporal region, and uneven enhancement. The dura mater and brain parenchyma are involved. T1 post-contrast (C,D) showed left sigmoid sinus filling defect.

multiple cerebral vessels (Figure 3). Mucor enters the cranium through the nasopharynx and paranasal sinuses, and typically manifests with vascular invasion. Cavernous sinus involvement can result in severe necrotizing vasculitis and thrombophlebitis of multiple large vessels and sinuses, and large vessel occlusion leading to a large area of cerebral infarction (Figure 7). Mucor can also cause mycotic aneurysms and hemorrhagic infarction.

\section{Conclusions}

Systemic and local infections can cause cerebrovascular complications. However, the clinical manifestations of cerebrovascular complications lack specificity and are highly variable. Diagnosis of cerebrovascular complications of infection relies on accurate clinical history and appropriate imaging evaluation. Cerebrovascular complications may have dramatic impact on treatment response and prognosis of patients, and may lead to poor prognosis, significant disability, and even death. Correct diagnosis has an important impact on the decision-making process and clinical management. Therefore, when imaging examination identifies patients with systemic or CNS infections and signs of cerebral infarction, cerebral hemorrhage, or cerebral 

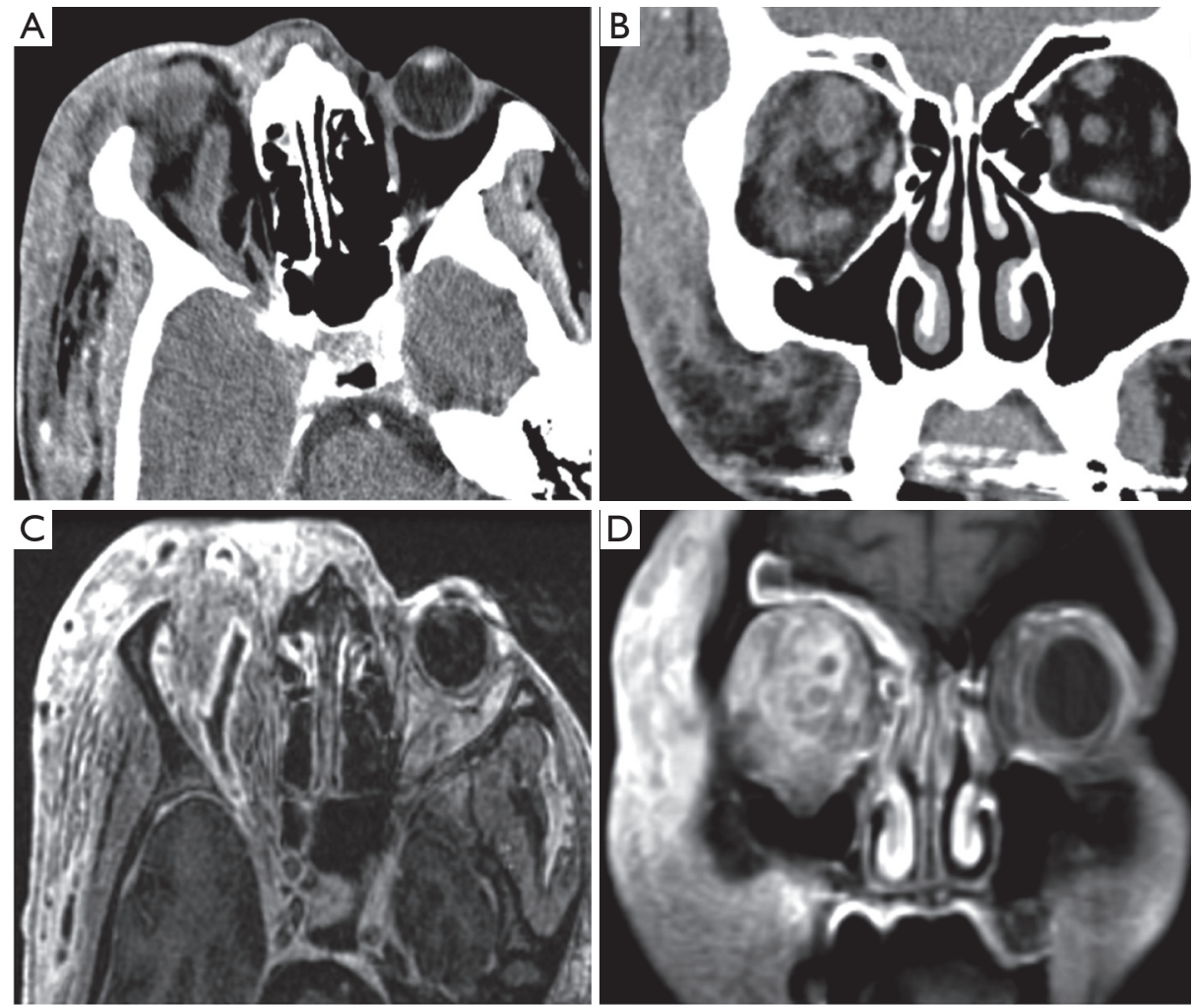

Figure 10 Right orbito-facial fungal cellulitis with thrombophlebitis. Axial (A) and coronal (B) CT showed extensive infectious lesions on the right side of the face and inside the eyelids. T1 post-contrast axial (C) and coronal (D) demonstrated ring enhancement in the right superior opthalmic vein. Infected lesions in the right eyelid and face showed a high signal. (The figure is provided by Fanny E. Morón MD, from the Department of Radiology, Baylor College of Medicine, Houston, Texas 77030, USA). CT, computed tomography.

ischemia, cerebrovascular complications of infection should be suspected.

\section{Acknowledgements}

Funding: This work was supported by the National Natural Science Foundation of China (grant No. 81871333 and 81471645).

\section{Footnote}

Conflicts of Interest: The authors have no conflicts of interest to declare.

\section{References}

1. Cantier M, Mazighi M, Klein I, Desilles JP, Wolff M, Timsit JF, Sonneville R. Neurologic complications of infective endocarditis: recent findings. Curr Infect Dis Rep
2017;19:41.

2. Kyaw H, Raju F, Shaikh AZ, Lin AN, Lin AT, Abboud J, Reddy S. Staphylococcus lugdunensis endocarditis and cerebrovascular accident: a systemic review of risk factors and clinical outcome. Cureus 2018;10:e2469.

3. Rohlwink UK, Kilborn T, Wieselthaler N, Banderker E, Zwane E, Figaji AA. Imaging features of the brain, cerebral vessels and spine in pediatric tuberculous meningitis with associated hydrocephalus. Pediatr Infect Dis J 2016; 35:e301-10.

4. Chow FC, Marra CM, Cho TA. Cerebrovascular disease in central nervous system infections. Semin Neurol 2011;31:286-306.

5. Guo Y, Bai Y, Yang C, Wang P, Gu L. Mycotic aneurysm due toSalmonellaspecies: clinical experiences and review of the literature. Braz J Med Biol Res 2018;51:e6864.

6. Ferfar Y, Savey L, Comarmond C, Sadaghianloo N, Garrido M, Domont F, Valantin MA, Pourcher-Martinez V, Cluzel P, Fouret P, Chiche L, Gaudric J, Koskas F, 
Cacoub P, Saadoun D. Large-vessel vasculitis in human immunodeficiency virus-infected patients. J Vasc Surg 2018;67:1501-11

7. Avcin T, Canova M, Guilpain P, Guillevin L, Kallenberg CG, Tincani A, Tonon M, Zampieri S, Doria A. Infections, connective tissue diseases and vasculitis. Clin Exp Rheumatol 2008;26:S18-26.

8. McKinney EF, Lee JC, Jayne DR, Lyons PA, Smith KG. T-cell exhaustion, co-stimulation and clinical outcome in autoimmunity and infection. Nature 2015;523:612-6.

9. Esfahani NZ, Anderson DM, Pieper C, Adams HP Jr. Intracerebral hemorrhage after IV tPA for stroke as early symptom of ANCA-associated vasculitis. eNeurologicalSci 2017;9:1-2.

10. Belizna CC, Hamidou MA, Levesque H, Guillevin L, Shoenfeld Y. Infection and vasculitis. Rheumatology (Oxford) 2009;48:475-82.

11. Clough RE, Taylor PR. Endovascular management of mycotic aortic aneurysms (review). Acta Chir Belg 2009;109:149-54.

12. Khatri IA, Wasay M. Septic cerebral venous sinus thrombosis. J Neurol Sci 2016;362:221-7.

13. Fugate JE, Lyons JL, Thakur KT, Smith BR, HedleyWhyte ET, Mateen FJ. Infectious causes of stroke. Lancet Infect Dis 2014;14:869-80.

14. Chow FC, Li Y, Hu Y, Chan J, Wang H, Xu W, Price RW, Sorond FA, Li T. Relationship between HIV infection, antiretroviral therapy, inflammatory markers and cerebrovascular endothelial function among adults in urban China. J Acquir Immune Defic Syndr 2017;74:339-46.

15. Schut ES, de Gans J, van de Beek D. Community-acquired bacterial meningitis in adults. Pract Neurol 2008;8:8-23.

16. Chow FC, Regan S, Zanni MV, Looby SE, Bushnell CD, Meigs JB, Grinspoon SK, Feske SK, Triant VA. Elevated ischemic stroke risk among women living with HIV infection. AIDS 2018;32:59-67.

17. Kastenbauer S, Pfister HW. Pneumococcal meningitis in adults: spectrum of complications and prognostic factors in a series of 87 cases. Brain 2003;126:1015-25.

18. Engelen-Lee JY, Brouwer MC, Aronica E, van de Beek D. Pneumococcal meningitis: Clinical-pathological correlations (meningene-path). Acta Neuropathol Commun 2016;4:26.

19. Mossa-Basha M, Hwang WD, De Havenon A, Hippe D, Balu N, Becker KJ, Tirschwell DT, Hatsukami T, Anzai Y, Yuan C. Multicontrast high-resolution vessel wall magnetic resonance imaging and its value in differentiating intracranial vasculopathic processes. Stroke 2015;46:1567-73.

20. Castro Caldas A, Geraldes R, Neto L, Canhão P, Melo TP. Central nervous system vasculitis associated with hepatitis $\mathrm{C}$ virus infection: a brain MRI-supported diagnosis. J Neurol Sci 2014;336:152-4.

21. Flores BC, Patel AR, Braga BP, Weprin BE, Batjer HH. Management of infectious intracranial aneurysms in the pediatric population. Childs Nerv Syst 2016;32:1205-17.

22. Singla A, Fargen K, Blackburn S, Neal D, Martin TD, Hess PJ, Beaver TM, Klodell CT, Hoh B. National treatment practices in the management of infectious intracranial aneurysms and infective endocarditis. J Neurointerv Surg 2016;8:741-6.

23. Matsubara N, Miyachi S, Izumi T, Yamanouchi T, Asai T, Ota K, Wakabayashi T. Results and current trends of multimodality treatment for infectious intracranial aneurysms. Neurol Med Chir (Tokyo) 2015;55:155-62.

24. Baddour LM, Wilson WR, Bayer AS, Fowler VG Jr, Bolger AF, Levison ME, Ferrieri P, Gerber MA, Tani LY, Gewitz MH, Tong DC, Steckelberg JM, Baltimore RS, Shulman ST, Burns JC, Falace DA, Newburger JW, Pallasch TJ, Takahashi M, Taubert KA; Committee on Rheumatic Fever, Endocarditis, and Kawasaki Disease; Council on Cardiovascular Disease in the Young; Councils on Clinical Cardiology, Stroke, and Cardiovascular Surgery and Anesthesia; American Heart Association; Infectious Diseases Society of America. Infective endocarditis: diagnosis, antimicrobial therapy, and management of complications: a statement for healthcare professionals from the Committee on Rheumatic Fever, Endocarditis, and Kawasaki Disease, Council on Cardiovascular Disease in the Young, and the Councils on Clinical Cardiology, Stroke, and Cardiovascular Surgery and Anesthesia, American Heart Association: endorsed by the Infectious Diseases Society of America. Circulation 2005;111:e394-434.

25. Ducruet AF, Hickman ZL, Zacharia BE, Narula R, Grobelny BT, Gorski J, Connolly ES Jr. Intracranial infectious aneurysms: a comprehensive review. Neurosurg Rev 2010;33:37-46.

26. Kannoth S, Thomas SV, Nair S, Sarma PS. Proposed diagnostic criteria for intracranial infectious aneurysms. J Neurol Neurosurg Psychiatry 2008;79:943-6.

27. Cho SM, Marquardt RJ, Rice CJ, Buletko AB, Zhang LQ, Khoury J, Thatikunta P, Hardman J, Wisco D, Uchino $\mathrm{K}$. Cerebral microbleeds predict infectious intracranial aneurysm in infective endocarditis. Eur J Neurol 
2018;25:970-5.

28. Zhou LX, Yao M, Cui LY, Li ML, Zhu YC, Ni J, Peng B. The structural imaging characteristics and its clinical relevance in patients with cerebral venous thrombosis-a retrospective analysis from one single center in China. Front Neurol 2017;8:648.

29. Bousser MG, Ferro JM. Cerebral venous thrombosis: an update. Lancet Neurol 2007;6:162-70.

30. Saposnik G, Barinagarrementeria F, Brown RD Jr, Bushnell CD, Cucchiara B, Cushman M, deVeber G, Ferro JM, Tsai FY; American Heart Association Stroke Council and the Council on Epidemiology and Prevention. Diagnosis and management of cerebral venous thrombosis: a statement for healthcare professionals from the American Heart Association/American Stroke Association. Stroke 2011;42:1158-92.

31. Linn J, Ertl-Wagner B, Seelos KC, Strupp M, Reiser M, Brückmann H, Brüning R. Diagnostic value of multidetector-row CT angiography in the evaluation of thrombosis of the cerebral venous sinuses. AJNR Am J Neuroradiol 2007;28:946-52.

32. Sabarudin A, Subramaniam C, Sun Z. Cerebral CT angiography and CT perfusion in acute stroke detection: a systematic review of diagnostic value. Quant Imaging Med Surg 2014;4:282-90.

Cite this article as: Shen G, Shen X, Pu W, Zhang G, Lerner A, Gao B. Imaging of cerebrovascular complications of infection. Quant Imaging Med Surg 2018;8(10):1039-1051. doi: 10.21037/ qims.2018.11.08 